\title{
UNITARY DILATION FOR POLAR DECOMPOSITIONS OF $p$-HYPONORMAL OPERATORS
}

\author{
MUNEO CHŌ \\ Department of Mathematics, Kanagawa University, Yokohama 221-8686, Japan \\ E-mail: chiyom01@kanagawa-u.ac.jp
}

TADASI HURUYA

Faculty of Education and Human Sciences, Niigata University, Niigata 950-2181, Japan

E-mail: huruya@ed.niigata-u.ac.jp

KÔTARÔ TANAHASHI

Department of Mathematics, Tohoku Pharmaceutical University, Sendai 981-8558, Japan

E-mail: tanahasi@tohoku-pharm.ac.jp

\begin{abstract}
In this paper, we introduce the angular cutting and the generalized polar symbols of a $p$-hyponormal operator $T$ in the case where $U$ of the polar decomposition $T=U|T|$ is not unitary and study spectral properties of it.
\end{abstract}

1. Introduction. As one of generalizations of normal operators, semi-hyponormal operators were introduced. In the study of semi-hyponormal operators, there exist many important techniques concerning polar decompositions $T=U|T|$ with unitary $U$ (for example, symbols, angular cutting and spectral mapping theorems). Many useful results have been obtained under the assumption that $U$ is unitary for the polar decomposition $T=U|T|$. Using a unitary dilation $\hat{U}$ of $U$ of a semi-hyponormal operator $T=U|T|$, we study spectral properties of the operator $U \varphi(|T|)$ for a semi-hyponormal operator $T=U|T|$ and a strictly monotone increasing continuous function $\varphi(\cdot)$.

In [8, Chapter 6, Section 3.2] Xia gave spectral mapping theorems of semi-hyponormal operators $T=U|T|$ with unitary $U$ for the following mapping: $T \rightarrow \xi(U) \varphi(|T|)$. In [7] Itoh extended these results to $p$-hyponormal operators. It is important for these results that

2000 Mathematics Subject Classification: Primary 47B20; Secondary 47A10.

Key words and phrases: Hilbert space, hyponormal operator, spectrum, angular cutting. Research of the first author supported by Grant-in-Aid Scientific Research No. 14540190. The paper is in final form and no version of it will be published elsewhere. 
$U$ is unitary. There exist important examples without this form (for example, unilateral weighted shift). In this paper we study spectral mapping theorems for a semi-hyponormal operator $T$ which does not have a polar decomposition $T=U|T|$ with unitary $U$.

Let $T$ be an operator in a Hilbert space $\mathcal{H}$. $T$ is said to be $p$-hyponormal if $\left(T^{*} T\right)^{p} \geq$ $\left(T T^{*}\right)^{p}$. If $p=1$ and $p=\frac{1}{2}$, then $T$ is said to be hyponormal and semi-hyponormal, respectively. By Löwner's Theorem, for $0<p<q \leq 1$, if $T$ is $q$-hyponormal, then $T$ is $p$-hyponormal. If $T$ is $p$-hyponormal, then $\operatorname{ker}(T) \subset \operatorname{ker}\left(T^{*}\right)$ (see [4]). Hence we may assume that $U$ is isometry if $T=U|T|$ is $p$-hyponormal. Let $\sigma(T), \sigma_{p}(T)$ and $\sigma_{a}(T)$ denote the spectrum, the point spectrum and the approximate point spectrum of $T$, respectively. Put $\sigma_{r}(T)=\sigma(T) \backslash \sigma_{a}(T)$. It is known from [8, Chapter 1, Section 3.1] that $\lambda \in \sigma_{r}(T)$ if and only if there exists a positive number $c$ such that $\|(T-\lambda I) x\| \geq c\|x\|(x \in \mathcal{H})$ and $(T-\lambda I)(\mathcal{H}) \neq \mathcal{H}$.

2. Results. Let $T=U|T|$ be an operator in a Hilbert space $\mathcal{H}$. If $U$ is isometry, let $\hat{U}=\left(\begin{array}{cc}U & I-U U^{*} \\ 0 & U^{*}\end{array}\right)$ and $\hat{T}=\hat{U}\left(\begin{array}{cc}|T| & 0 \\ 0 & 0\end{array}\right)$. Then we have $|\hat{T}|=|T| \oplus 0$. If $T=U|T|$ is $p$-hyponormal, then we assume that $U$ is isometry and hence we can define $\hat{U}$ and $\hat{T}$.

Proposition 1. Let $T=U|T|$ be an operator in a Hilbert space $\mathcal{H}$. If there exists a unitary operator $\hat{U}$ on $\mathcal{H} \oplus \mathcal{H}$ such that $\hat{U}(x \oplus 0)=U x \oplus 0$, then the following equalities hold for $T$ and $\hat{T}=\hat{U}(|T| \oplus 0)$ :

(1) $\sigma(T) \backslash\{0\}=\sigma(\hat{T}) \backslash\{0\}$,

(2) $\sigma_{a}(T) \backslash\{0\}=\sigma_{a}(\hat{T}) \backslash\{0\}$,

(3) $\sigma_{r}(T) \backslash\{0\}=\sigma_{r}(\hat{T}) \backslash\{0\}$.

Proof. The proof of (1) is very similar to that of (3). Hence there is no need to cite [8, Chapter 2, Lemma 3.5]. If (3) holds, then so does (2). It is sufficient to show that (3) holds. If $\lambda \in \sigma_{r}(T) \backslash\{0\}$, there exists $c>0$ such that $\|(T-\lambda I) x\| \geq c\|x\|(x \in \mathcal{H})$ and $(T-\lambda I)(\mathcal{H}) \neq \mathcal{H}$. Then

$$
\|(\hat{T}-\lambda I)(x \oplus y)\|^{2}=\|(T-\lambda I) x\|^{2}+\|-\lambda y\|^{2} \geq c_{1}^{2}\|x \oplus y\|^{2}
$$

where $c_{1}=\min \{c,|\lambda|\}$. Since $(T-\lambda I)(\mathcal{H}) \neq \mathcal{H}$, it is easy to check $(\hat{T}-\lambda I)(\mathcal{H} \oplus \mathcal{H}) \neq$ $\mathcal{H} \oplus \mathcal{H}$, so that $\lambda \in \sigma_{r}(\hat{T})$.

Conversely if $\lambda \in \sigma_{r}(\hat{T}) \backslash\{0\}$, then there exists $c>0$ such that $\|(\hat{T}-\lambda I)(x \oplus y)\| \geq$ $c\|x \oplus y\|(x \oplus y \in \mathcal{H} \oplus \mathcal{H})$ and $(\hat{T}-\lambda I)(\mathcal{H} \oplus \mathcal{H}) \neq \mathcal{H} \oplus \mathcal{H}$. Then

$$
\|(T-\lambda I) x\|=\|(\hat{T}-\lambda I)(x \oplus 0)\| \geq c\|x \oplus 0\|=c\|x\| .
$$

Suppose that $(T-\lambda I)(\mathcal{H})=\mathcal{H}$. For $x, y \in \mathcal{H}$, there exists $x_{1} \in \mathcal{H}$ such that $(T-\lambda I) x_{1}=$ $x$, so that

$$
x \oplus y=(\hat{T}-\lambda I)\left(x_{1} \oplus-(1 / \lambda) y\right) .
$$

This is a contradiction. Hence $\lambda \in \sigma_{r}(T)$.

TheOREM 2. Let $T=U|T|$ be a semi-hyponormal operator and $\varphi$ be a strictly monotone increasing continuous function on $[0,\|T\|]$. If $\varphi$ is operator monotone on $[0,\|T\|]$ and 
$\varphi(0)=0$, then

$$
\begin{aligned}
\sigma(U \varphi(|T|)) & =\left\{e^{i \theta} \varphi(r): e^{i \theta} r \in \sigma(U|T|)\right\}, \\
\sigma_{a}(U \varphi(|T|)) & =\left\{e^{i \theta} \varphi(r): e^{i \theta} r \in \sigma_{a}(U|T|)\right\}, \\
\sigma_{r}(U \varphi(|T|)) & =\left\{e^{i \theta} \varphi(r): e^{i \theta} r \in \sigma_{r}(U|T|)\right\} .
\end{aligned}
$$

Proof. If $0 \notin \sigma(T)$, then $T=U|T|$ with unitary $U$. In this case, the result follows from [8, Chapter 6 , Theorem 3.2]. We assume that $0 \in \sigma(T)$. Since $\varphi$ is operator monotone and $\hat{U}|\hat{T}| \hat{U}^{*}=\left|\hat{T}^{*}\right| \leq|\hat{T}|$, we get $\hat{U}^{*} \varphi(|\hat{T}|) \hat{U}=\varphi\left(\hat{U}^{*}|\hat{T}| \hat{U}\right) \leq \varphi(|\hat{T}|)$ which implies that $\hat{U} \varphi(|\hat{T}|)$ is semi-hyponormal. By Xia's theorem [8, Chapter 6, Theorem 3.2], we again have

$$
\sigma_{*}(\hat{U} \varphi(|\hat{T}|))=\left\{e^{i \theta} \varphi(r): e^{i \theta} r \in \sigma_{*}(\hat{U}|\hat{T}|)\right\},
$$

where $\sigma_{*}$ denotes $\sigma, \sigma_{a}$ or $\sigma_{r}$. By Proposition 1 ,

$$
\sigma_{*}(\hat{U} \varphi(|\hat{T}|)) \backslash\{0\}=\sigma_{*}(U \varphi(|T|)) \backslash\{0\}
$$

and

$$
\sigma_{*}(\hat{U}|\hat{T}|) \backslash\{0\}=\sigma_{*}(U|T|) \backslash\{0\}
$$

Therefore, we have

$$
\sigma_{*}(U \varphi(|T|)) \backslash\{0\}=\left\{e^{i \theta} \varphi(r): e^{i \theta} r \in \sigma_{*}(U|T|)\right\} \backslash\{0\} .
$$

Next since $U$ satisfies $\|U|T| x\|=\||T| x\|$ for any vector $x$, we have

$$
0 \in \sigma_{a}(U|T|) \text { if and only if } 0 \in \sigma_{a}(U \varphi(|T|)) .
$$

Since $\sigma_{r}(T)=\sigma(T) \backslash \sigma_{a}(T)$, we have

$$
0 \in \sigma_{r}(U|T|) \text { if and only if } 0 \in \sigma_{r}(U \varphi(|T|)) .
$$

This completes the proof.

By comparison in case of hyponormal operators [2], the following example implies that in the semi-hyponormal case, spectral mapping theorems are complicated.

EXAmple. Let $\left\{e_{k}\right\}_{k=1}^{\infty}$ be the canonical orthnormal basis for the Hilbert space $l^{2}$. Let $U$ be the unilateral shift.

Let $T=U$. Then $T$ is hyponormal and $|T|=I$, so that $T$ is semi-hyponormal. Let $\varphi$ be a function on $\mathbf{R}$ such that $\varphi(x)=x+1$. We remark $\varphi(0)=1$. Then $U \varphi(I)=2 U$, so that $U \varphi(I)$ is semi-hyponormal. Therefore, $\sigma(U \varphi(I))=\{z:|z| \leq 2\}$ and $\left\{e^{i \theta} \varphi(r)\right.$ : $\left.r \cdot e^{i \theta} \in \sigma(T)\right\}=\{z: 1 \leq|z| \leq 2\}$, so that $\sigma(U \varphi(|T|)) \neq\left\{e^{i \theta} \varphi(r): r e^{i \theta} \in \sigma(U|T|)\right\}$.

Definition 1. Let $T=U|T|$ be a semi-hyponormal operator such that $U$ is not unitary. Let $\mathbf{T}=\{z:|z|=1\}$ be the unit circle. For a unitary operator $\hat{U}$ on $\mathcal{K}=\mathcal{H} \oplus \mathcal{H}$, let $\hat{U}=\int_{\mathbf{T}} \lambda d E(\lambda)$ be the spectral decomposition $\hat{U}$. Let $\gamma$ be an arc in $\mathbf{T}$ and $E(\gamma) \neq 0$. For $\hat{T}=\hat{U}|\hat{T}|$, denote

$$
\mathcal{K}_{\gamma}=E(\gamma) \mathcal{K}, \quad \hat{U}_{\gamma}=\hat{U}_{\mid \mathcal{K}_{\gamma}} \text { and } \hat{T}_{\gamma}=E(\gamma) \hat{T}_{\mid \mathcal{K}_{\gamma}} .
$$

$\hat{T}_{\gamma}$ is called the section of $\hat{T}$ cut by the arc $\gamma$. We denote $\hat{T}_{\gamma}$ simply by $T_{\gamma}$. 
THEOREM 3. Let $T=U|T|$ be a semi-hyponormal operator such that $U$ is not unitary. Let $\gamma$ be an arc in the unit circle $\mathbf{T}$ with $E(\gamma) \neq 0$. Put $\mathcal{D}_{\gamma}=\{\lambda: \lambda \neq 0, \lambda /|\lambda| \in \gamma\}$. Then

$$
\sigma_{p}\left(T_{\gamma}\right) \backslash\{0\}=\sigma_{p}(T) \cap \mathcal{D}_{\gamma},
$$

in addition, $x$ is an eigenvector of $T_{\gamma}$ corresponding to the eigenvalue $\lambda$ if and only if $x$ is an eigenvector of $T$ and $\lambda \in \mathcal{D}_{\gamma}$,

$$
\sigma\left(T_{\gamma}\right) \subset \overline{\mathcal{D}}_{\gamma} \cap \sigma(T)
$$

moreover, if $\gamma$ is an open arc, then

$$
\begin{aligned}
& \sigma_{a}\left(T_{\gamma}\right) \cap \mathcal{D}_{\gamma}=\sigma_{a}(T) \cap \mathcal{D}_{\gamma}, \\
& \sigma_{r}\left(T_{\gamma}\right) \cap \mathcal{D}_{\gamma}=\sigma_{r}(T) \cap \mathcal{D}_{\gamma},
\end{aligned}
$$

thus

$$
\sigma\left(T_{\gamma}\right) \cap \mathcal{D}_{\gamma}=\sigma(T) \cap \mathcal{D}_{\gamma}
$$

Proof. By [8, Chapter 6, Theorem 3.9], $\sigma\left(T_{\gamma}\right) \subset \sigma(\hat{T})=\sigma(T)$. An application of [8, Chapter 1, Theorem 3.2] and Proposition 1 complete the proof.

REMARK. For an open arc $\gamma, \sigma_{*}\left(T_{\gamma}\right) \cap \mathcal{D}_{\gamma}$ is independent of the choice of a unitary operator $\hat{U}$, where $\sigma_{*}$ is $\sigma, \sigma_{a}$ or $\sigma_{r}$.

For a unitary operator $U$ and an operator $A$, if

$$
\mathcal{S}_{U}^{ \pm}(A)=\mathrm{s}-\lim _{n \rightarrow \pm \infty} U^{-n} A U^{n}
$$

exist, then $\mathcal{S}_{U}^{ \pm}(A)$ are called the general polar symbols of $A$ related to $U$. If $T=U|T|$ is semi-hyponormal with unitary $U$, then $\mathcal{S}_{U}^{ \pm}(|T|)$ exist (cf. [8]). For $0 \leq k \leq 1$, we define

$$
T_{k}=U\left(k \mathcal{S}_{U}^{+}(|T|)+(1-k) \mathcal{S}_{U}^{-}(|T|)\right) .
$$

The operator $T_{k}$ is called the generalized polar symbol of $T$ with respect to $k$. In case $U$ is not unitary, let $\hat{U}$ be the unitary dilation of $U$. For operators $\hat{U}$ and $\hat{T}$, since $\hat{T}=\hat{U}|\hat{T}|$ is semi-hyponormal with unitary $\hat{U}$, there exists $\mathcal{S}_{\hat{U}}^{ \pm}(|\hat{T}|)$. For $0 \leq k \leq 1$, we also define

$$
\hat{T}_{k}=\hat{U}\left(k \mathcal{S}_{\hat{U}}^{+}(|\hat{T}|)+(1-k) \mathcal{S}_{\hat{U}}^{-}(|\hat{T}|)\right) .
$$

Then Xia proved the following.

Theorem A ([8, Chapter 4, Theorem 4.1]). Let $T=U|T|$ be semi-hyponormal with unitary $U$. Then

$$
\sigma(T)=\bigcup_{0 \leq k \leq 1} \sigma\left(T_{k}\right) .
$$

We have the following result.

TheOREM 4. Let $T=U|T|$ be semi-hyponormal such that $U$ is not unitary. Then

$$
\sigma(T)=\bigcup_{0 \leq k \leq 1} \sigma\left(\hat{T}_{k}\right)
$$


Proof. Since $\hat{T}=\hat{U}\left(\begin{array}{cc}|T| & 0 \\ 0 & 0\end{array}\right)$ is semi-hyponormal with unitary $\hat{U}$, by Theorem A we have

$$
\sigma(\hat{T})=\bigcup_{0 \leq k \leq 1} \sigma\left(\hat{T}_{k}\right) .
$$

Since $U$ is not unitary, $\sigma(T)=\sigma(\hat{T})$. Hence the proof is complete.

Let $T=U|T|$ is $p$-hyponormal with $U$ which is not unitary. If $S=U|T|^{2 p}$, then $S$ is semi-hyponormal. Let $|\hat{S}|=\left(\begin{array}{cc}|T|^{2 p} & 0 \\ 0 & 0\end{array}\right)$. Then we can define the semi-hyponormal operator $\hat{S}=\hat{U}|\hat{S}|$ with unitary $\hat{U}$.

TheOREM 5. Let $T=U|T|$ be a p-hyponormal operator such that $U$ is not unitary. Let $S=U|T|^{2 p}$ and $\hat{S}=\hat{U}\left(\begin{array}{cc}|T|^{2 p} & 0 \\ 0 & 0\end{array}\right)$. Then $\mathcal{S}_{\hat{U}}^{ \pm}(|\hat{S}|)$ exist and

$$
\sigma(T)=\bigcup_{0 \leq k \leq 1} \sigma\left(T_{[k]}\right),
$$

where

$$
T_{[k]}=\hat{U}\left(k \mathcal{S}_{\hat{U}}^{+}(|\hat{S}|)+(1-k) \mathcal{S}_{\hat{U}}^{-}(|\hat{S}|)\right)^{\frac{1}{2 p}} .
$$

Proof. Since $\hat{S}=\hat{U}\left(\begin{array}{cc}|T|^{2 p} & 0 \\ 0 & 0\end{array}\right)$ is semi-hyponormal with unitary $\hat{U}$, from Theorem A it follows that

$$
\sigma(\hat{S})=\bigcup_{0 \leq k \leq 1} \sigma\left(\hat{S}_{k}\right) .
$$

Proposition 1 implies $\sigma(S)=\sigma(\hat{S})$. Since by Theorem 3 of [5]

$$
\sigma(S)=\left\{r^{2 p} e^{i \theta}: r e^{i \theta} \in \sigma(T)\right\}
$$

it follows that $r e^{i \theta} \in \sigma(T)$ if and only if $r^{2 p} e^{i \theta} \in \bigcup_{0 \leq k \leq 1} \sigma\left(\hat{S}_{k}\right)$. Since

$$
\hat{S}_{k}=\hat{U}\left(k \mathcal{S}_{\hat{U}}^{+}(|\hat{S}|)+(1-k) \mathcal{S}_{\hat{U}}^{-}(|\hat{S}|)\right)
$$

is normal, it follows that $r^{2 p} e^{i \theta} \in \sigma\left(\hat{S}_{k}\right)$ if and only if $r e^{i \theta} \in \sigma\left(T_{[k]}\right)$. So the proof is complete.

Finally, we introduce Xia spectrum for a $p$-hyponormal operator $T=U|T|$ such that $U$ is not unitary.

For a pair $(A, B)$ of operators, $(z, w) \in \mathbf{C}^{2}$ belongs to the joint approximate point spectrum $\sigma_{j a}(A, B)$ if there exists a sequence $\left\{x_{n}\right\}$ of unit vectors such that $(A-z) x_{n} \rightarrow 0$ and $(B-w) x_{n} \rightarrow 0$ as $n \rightarrow \infty$. It is well known that if $(A, B)$ is a commuting pair, then $\sigma_{j a}(A, B)$ is non-empty (cf. [1, Proposition 2]).

Definition 2. Let $T=U|T|$ be a $p$-hyponormal operator such that $U$ is not unitary. Let $S=U|T|^{2 p}, \hat{S}=\hat{U}\left(\begin{array}{cc}|T|^{2 p} & 0 \\ 0 & 0\end{array}\right)$ and

$$
\left|T_{[k]}\right|=\left(k \mathcal{S}_{\hat{U}}^{+}(|\hat{S}|)+(1-k) \mathcal{S}_{\hat{U}}^{-}(|\hat{S}|)\right)^{\frac{1}{2 p}} .
$$


Since $\hat{U}$ commutes with every $\left|T_{[k]}\right|(0 \leq k \leq 1)$, we see that $\sigma_{j a}\left(\hat{U},\left|T_{[k]}\right|\right)$ is non-empty. We define the Xia spectrum $\sigma_{X}(T)$ of $T$ by

$$
\sigma_{X}(T)=\bigcup_{0 \leq k \leq 1} \sigma_{j a}\left(\hat{U},\left|T_{[k]}\right|\right) .
$$

The following corollary is a direct consequence of Theorem 5 and the fact that $r e^{i \theta} \in$ $\sigma\left(T_{[k]}\right)$ iff $\left(e^{i \theta}, r\right) \in \sigma_{j a}\left(\hat{U},\left|T_{[k]}\right|\right)$. So we omit the proof.

Corollary 6. Let $T=U|T|$ be a p-hyponormal operator such that $U$ is not unitary. Let $S=U|T|^{2 p}, \hat{S}=\hat{U}\left(\begin{array}{cc}|T|^{2 p} & 0 \\ 0 & 0\end{array}\right)$ and

$$
\left|T_{[k]}\right|=\left(k \mathcal{S}_{\hat{U}}^{+}(|\hat{S}|)+(1-k) \mathcal{S}_{\hat{U}}^{-}(|\hat{S}|)\right)^{\frac{1}{2 p}} .
$$

Then $r e^{i \theta} \in \sigma(T)$ if and only if $\left(e^{i \theta}, r\right) \in \sigma_{X}(T)$.

Acknowledgments. The authors express their thanks to the referee for his useful suggestions.

\section{References}

[1] J. Bunce, The joint spectrum of commuting nonormal operators, Proc. Amer. Math. Soc. 29 (1971), 499-505.

[2] M. Chō, Gigga, Y. M. Han and T. Huruya, Spectral mapping theorems for hyponormal operators, Proc. Royal Irish Acad., to appear.

[3] M. Chō and T. Huruya, $p$-Hyponormal operators for $0<p<1 / 2$, Comment. Math. Prace Mat. 33 (1993), 23-29.

[4] M. Chō and T. Huruya, Putnam's inequality for p-hyponormal n-tuples, Glasgow Math. J. 41 (1999), 13-17.

[5] M. Chō and M. Itoh, Putnam's inequality for p-hyponormal operators, Proc. Amer. Math. Soc. 123 (1995), 2435-2440.

[6] M. Chō and M. Itoh, On spectra of p-hyponormal operators, Integral Equations and Operator Theory 23 (1995), 287-293.

[7] M. Itoh, Spectral mapping theorems for p-hyponormal operators, Acta Sci. Math. (Szeged) 62 (1996), 523-535.

[8] D. Xia, Spectral Theory of Hyponormal Operators, Birkhäuser Verlag, Basel, 1983. 